\title{
Rosette-Forming Glioneuronal Tumor: A Rare Posterior Fossa Tumor in an Old Adult
}

\author{
Jitin Goyal ${ }^{1} \quad$ Ankush Jajodia ${ }^{1} \quad$ Anila Sharma ${ }^{2}$ Sunil Pasricha ${ }^{2}$ Sunil Kumar Puri ${ }^{1}$
}

1Department of Radiology, Rajiv Gandhi Cancer Hospital and Research Institute, Delhi, India

2Department of Histopathology, Rajiv Gandhi Cancer Hospital and Research Institute, Delhi, India

Ind J Med Paediatr Oncol 2021;42:282-283.

\section{Introduction}

Rosette-forming glioneuronal tumor (RGNT) is an uncommon, slow growing low-grade (World Health Organization [WHO] grade I) glioneuronal type of primary brain tumor. It was included as a distinct glioneuronal neoplasm in the 2016 WHO classification of tumors of the central nervous system. ${ }^{1,2}$ Initially, it was described as dysembryoplastic neuroepithelial tumor of cerebellum. ${ }^{3}$ RGNTs more often occur in young adults with mean age of 23.5 years with a female predominance. ${ }^{1,4}$ Patient presents clinically with headache, which is the most common symptom and clinical manifestations of raised intracranial pressure or ataxia, depending on the location of the tumor. The primary treatment modality is surgery and gross total resection is almost curative. 4,5

A 55-year-old male presented in our hospital with complaints of headache for the last 6 months. Magnetic resonance evaluation study revealed a solid cystic lesion with hyperintense cystic component and intermediate-hypointense solid component on T2 axial images with no evidence of perilesional edema, centered in posterior fossa, involving midline vermis without any significant mass effect on cerebellar structures. The lesion showed few foci of blooming on gradient sequences that appeared as bright foci on corresponding phase sequences, likely suggestive of calcification. On post-contrast sequences, the solid component in lesion showed enhancing nodular component.

Sagittal post-contrast sequences showed mild mass effect on the fourth ventricle with concomitant expansion and upstream dilatation of bilateral lateral and third ventricles suggestive of early onset hydrocephalus ( - Fig. 1).

Surgical intervention in the form of gross total resection was planned and the patient underwent suboccipital craniotomy with tumor decompression and external ventricular drain placement.

Intraoperative findings showed a solid cystic tumor, whitish in appearance arising from the fourth ventricle.
Address for correspondence Ankush Jajodia, Department of Radiology, Rajiv Gandhi Cancer Hospital and Research Institute, Sector 5; Rohini 110085 Delhi, India (e-mail: ankushjaj@gmail.com).
Microscopically, the tumor was moderately cellular and had a biphasic neurocystic and glial architecture. The neurocystic component had ring-shaped neurocystic rosettes around eosinophilic neuropil cores. The neurocystic tumor cells had

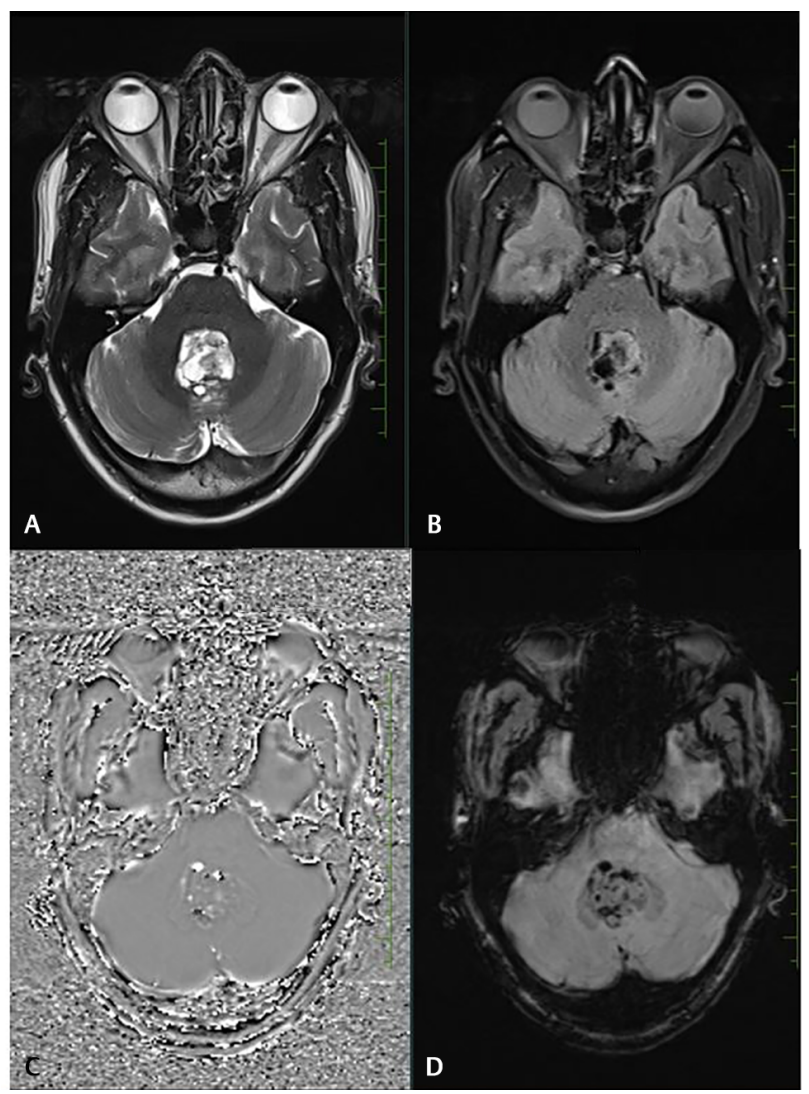

Fig. 1 (A) Axial T2-weighted and (B) fluid-attenuated inversion recovery image shows heterogeneous solid-cystic mass centered in midline involving vermis in posterior fossa, with no perilesional edema. (C and D) Axial gradient sequences show foci of blooming that appears as bright foci on corresponding phase sequences, suggestive of calcification.
DOI https://doi.org/

$10.1055 / \mathrm{s}-0041-1735761$ ISSN 0971-5851
(C) 2021. Indian Society of Medical and Paediatric Oncology.

This is an open access article published by Thieme under the terms of the Creative Commons Attribution-NonDerivative-NonCommercial-License, permitting copying and reproduction so long as the original work is given appropriate credit. Contents may not be used for commercial purposes, or adapted, remixed, transformed or built upon. (https://creativecommons.org/licenses/by-nc-nd/4.0/).

Thieme Medical and Scientific Publishers Private Ltd. A-12, Second Floor, Sector -2, NOIDA -201301, India 


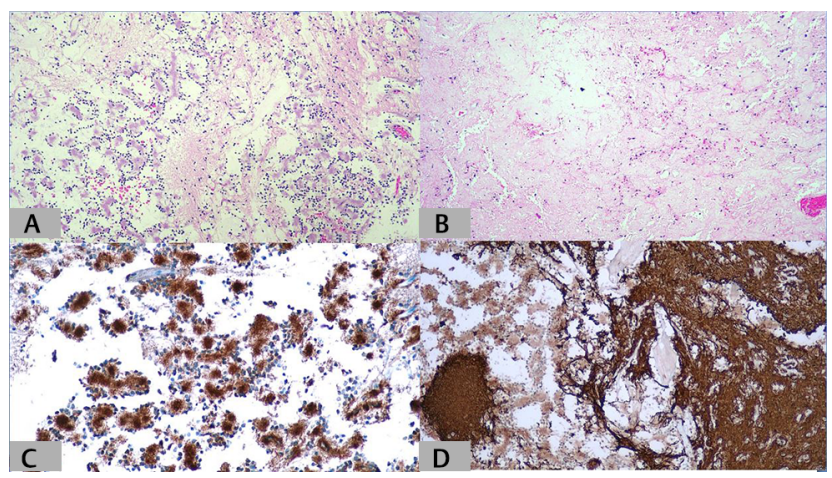

Fig. 2 (A) HPE: Hematoxylin and eosin (H \& E), Rosettes are having ring-shaped array of neurocytic nuclei around delicate eosinophilic neuropil cores. Original magnification x100. (B) HPE: (H \& E) Spindle-shaped astrocytic cells in myxoid background. Original magnification x100. (C) Immunohistochemistry: Synaptophysin positivity in center of rosettes. Original magnification $\times 100$. (D) Immunohistochemistry: Glial fibrillary acidic protein positivity in glial component and negative in neurocytic component. Original magnification $\times 100$.

spherical nuclei with abundant cytoplasm in myxoid background. The glial component showed spindle to stellate shaped nuclei with dense chromatin in a fibrillary background and focal occurrence of Rosenthal fibers with hemosiderin deposits. No necrosis or calcification was seen. On immunohistochemistry (IHC), synaptophysin strongly highlighted center of rosettes. S-100 was positive in tumor cells. Glial fibrillary acidic protein was positive in glial component. $\mathrm{K}_{\mathrm{i}}-67$ index was $1 \%$ ( - Fig. 2). Diagnosis of RGNT (WHO grade I) was proposed based on these characteristic pathological features.

\section{Discussion}

Radiologically, RGNT has been described as a wellcircumscribed solid-cystic lesion. The imaging differentials of RGNT at this location include pilocytic astrocytoma, ependymomas, metastasis, and medulloblastoma, but age excludes all except metastasis and ependymomas.
Cystic changes with hemorrhage are more common in ependymomas and multiple lesions with systemic complaints in other organs may give a clue to metastasis. Pathologically in cases where neurocytic components are not appreciated or not biopsied, RGNT may be misdiagnosed as pilocytic astrocytoma pathologically. The absence of pseudorosettes differentiates pilocytic astrocytoma from RGNT. Definitive diagnosis is by IHC. ${ }^{6}$ RGNTs are benign tumors with favorable prognosis and usually are treated by surgical resection only.

\section{Declaration of Patient Consent}

The authors certify that they have obtained all appropriate patient consent forms.

\section{Conflicts of Interest}

The authors declare that there are no known conflicts of interest.

\section{References}

1 Louis DN, Perry A, Reifenberger G, et al. The 2016 World Health Organization Classifcation of Tumors of the Central Nervous System: a summary. Acta Neuropathol 2016;131(6):803-820

2 Schlamann A, von Bueren AO, Hagel C, et al. An individual patient data meta-analysis on characteristics and outcome of patients with papillary glioneuronal tumor, rosette glioneuronal tumor with neuropil-like islands and rosette forming glioneuronal tumor of the fourth ventricle. PLoS One 2014;9(7):e101211

3 Kuchelmeister K, Demirel T, Schlörer E, Bergmann M, Gullotta F. Dysembryoplastic neuroepithelial tumour of the cerebellum. Acta Neuropathol 1995;89(4):385-390

4 Anyanwu CT, Robinson TM, Huang JH. Rosette-forming glioneuronal tumor: an update. Clinical and Translational Oncology. 2020;22(5):623-30

5 Matsumura N, Wang Y, Nakazato Y. Coexpression of glial and neuronal markers in the neurocytic rosettes of rosette-forming glioneuronal tumors. Brain Tumor Pathol 2014;31(1):17-22

6 Pradhan R, Mondal S, Pal S, Chatterjee S, Banerjee A, Bhattacharyya D. Rosette-forming glioneuronal tumor of the fourth ventricle. Neurol India 2017;65(5):1176 